\title{
A Case of Appendiceal Goblet Cell Carcinoid Tumor: Getting it right under the Microscope
}

\author{
Waye Hann Kang, ${ }^{1}$ Norasyikin Abdul Wahab, ${ }^{2}$ Nor Azmi Kamaruddin ${ }^{2}$ \\ ${ }^{1}$ Faculty of Medicine and Health Sciences, University Tunku Abdul Rahman, Malaysia \\ ${ }^{2}$ Department of Medicine, Pusat Perubatan Universiti Kebangsaan Malaysia
}

\begin{abstract}
Goblet cell carcinoid (GCC) is a rare neoplasm of the vermiform appendix and can be mistaken as a typical neuroendocrine tumour (TNET). The natural history of this disease is more aggressive compared to TNETs and requires a more aggressive approach. We report a case of a 37-year-old male who was initially diagnosed with TNET, but subsequently revised as Tang's A GCC. He underwent appendectomy and right hemicolectomy. Aside from a persistently elevated carcinoembyrogenic antigen (CEA) result, his ${ }^{18} \mathrm{~F}$-fluorodeoxyglucose (FDG) $\mathrm{PET} / \mathrm{CT}$ and a 68-Gallium DOTATATE PET/CT scan showed no FDG or DOTATATE avid lesions.
\end{abstract}

Key words: goblet cell carcinoid, mixed neuroendocrine-non-endocrine neoplasm, MiNEN, histopathology

\section{INTRODUCTION}

Goblet cell carcinoid (GCC) is a rare neoplasm of the vermiform appendix, and is often diagnosed in less than $1 \%$ of appendectomy specimens. ${ }^{1,2}$ They are often diagnosed incidentally during a histopathological examination after an appendectomy for "suspected appendicitis." GCCs often present as classical acute appendicitis (right iliac fossa pain and tenderness), or even as vague abdominal complaints. GCCs present more commonly in the 50-60 years old age group, with no gender predilection, and a majority of the cases are reported in the Caucasian population. Distinctive histopathological features of a GCC are the presence of both goblet cells (glandular components) and neuroendocrine components. Due to its rarity, it may be misinterpreted as a neuroendocrine tumor. This case describes a patient who was initially diagnosed as TNET, but later the diagnosis was revised as GCC and underwent a more aggressive management approach.

\section{CASE}

A 37-year-old healthy male presented to a private hospital with right iliac fossa pain and was diagnosed to have acute appendicitis. He underwent a laparoscopic appendectomy uneventfully and the histopathological examination of the appendix was reported as a neuroendocrine tumour of the appendix with lymphovascular infiltration.

The patient denied symptoms suggesting of a carcinoid tumour, such as diarrhoea, flushing or wheezing. He denies any constitutional symptoms and there was no family history of malignancy. Clinical examination was uneventful. He was subsequently referred to our centre for further evaluation.

Revised histopathological examination (HPE) showed mixed neuroendocrine-non-neuroendocrine neoplasm (MiNEN) of intermediate grade (goblet cell carcinoid) as there were two morphologically distinct tumour components. The conventional group, composing of nests and cords of tumour cells, exhibited round uniform nuclei with salt and pepper chromatin and scanty eosinophilic cytoplasm (NET appearance). The second group of cells displayed irregular infiltrating nests of goblet cells with abundant univacuolated cytoplasm and peripheral compressed nuclei. Both tumour groups (Figures 1 and 2) encompass $>30 \%$ of tumour volume each. Mitotic figures were not seen. Immunohistochemical studies showed both tumour groups being positive to synaptophysin and chromogranin. Only the goblet group shows positivity to CK20. The Ki-67 proliferation index is $<2 \%$ (Figure 3 ).

Based on the HPE results, he was classified as Tang's classification group A (classic GCC and underwent right hemicolectomy. The histopathological examination of the colon and its surrounding lympho-vascular are showed no local invasion.

His whole body ${ }^{18} \mathrm{~F}$-fluorodeoxyglucose (FDG) PET/ CT and a 68-Gallium DOTATATE PET/CT scan showed no FDG or DOTATATE avid lesions. His post-operative CEA was elevated despite his being asymptomatic. At the moment the patient is still under close surveillance with routine CEA measurement and FDG PET/CT Scan.
ISSN 0857-1074 (Print) | eISSN 2308-118x (Online)

Printed in the Philippines

Copyright (C) 2020 by Kang et al.

Received: February 19, 2020. Accepted: April 1, 2020.

Published online first: April 19, 2020.

https://doi.org/10.15605/jafes.035.01.16
Corresponding author: Waye Hann Kang, MD, MRCP(UK)

Lecturer/Endocrinology Trainee

Faculty of Medicine and Health Sciences, University Tunku Abdul Rahman,

Lot PT 21144, Jalan Sungai Long, Bandar Sungai Long,

43000 Kajang, Selangor, Malaysia

Tel. No.: +60390860288

Fax No.: +60390198868

E-mail: kangwh@utar.edu.my

ORCiD: https://orcid.org/0000-0003-3209-7196 


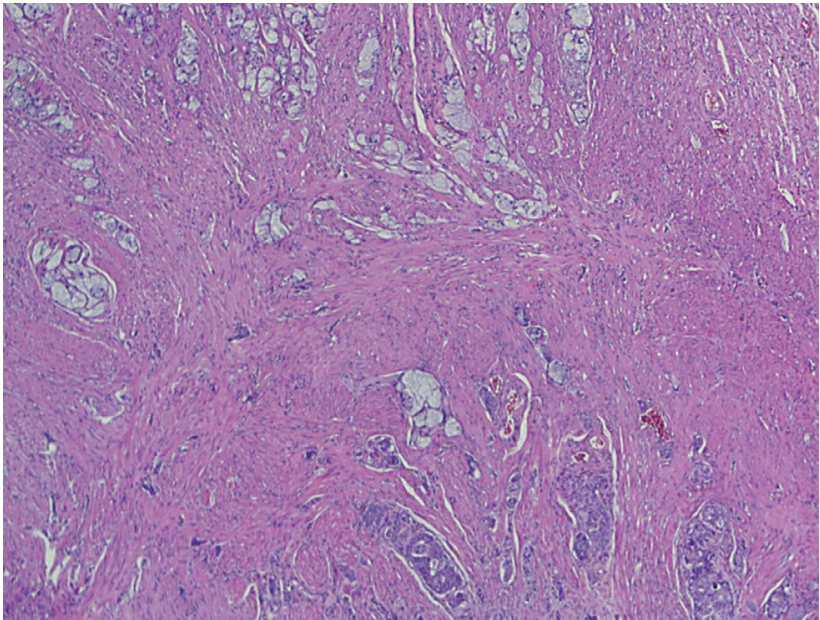

Figure 1. Microscopic findings of the resected appendix showed tumour cells infiltration into the muscularis propriae, with two distinct components identified (H\&E, 40x).

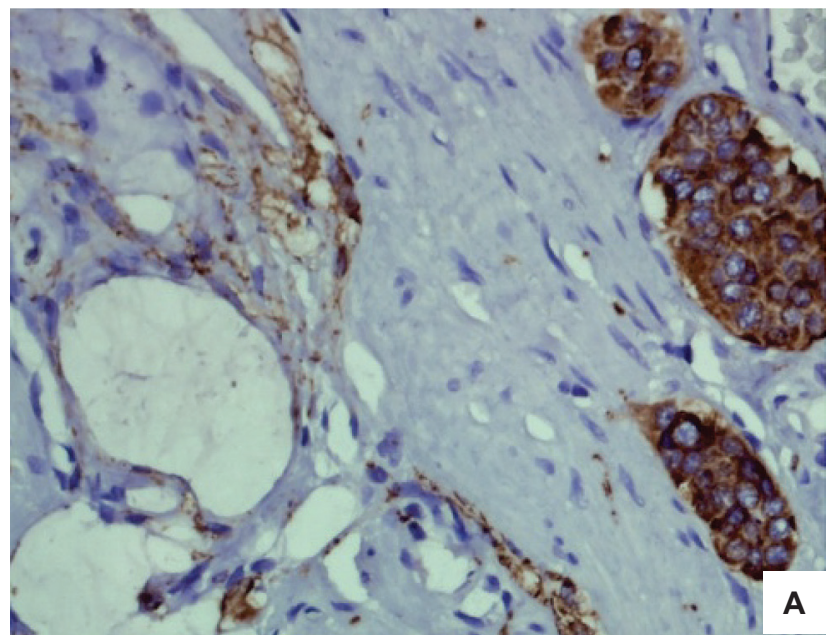

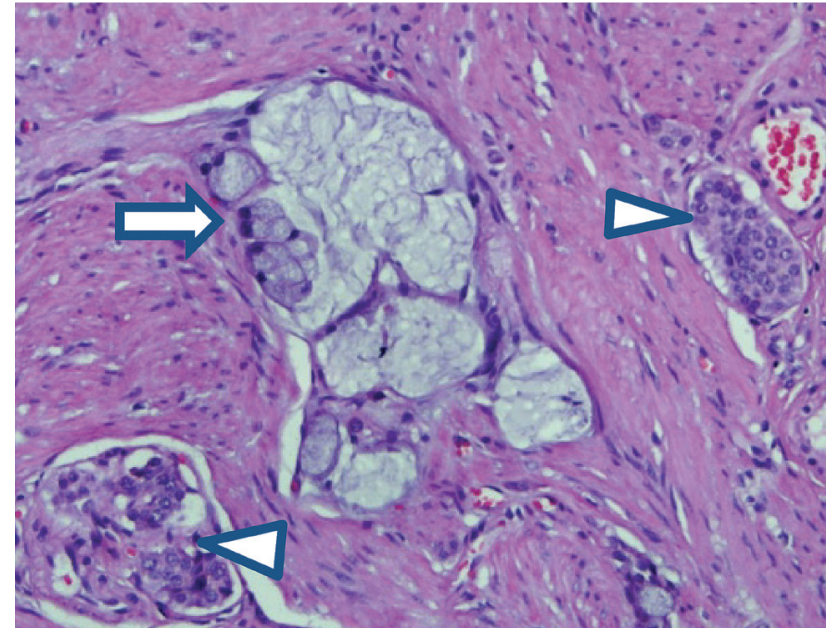

Figure 2. First component: nests of tumour cells exhibiting uniform, round nuclei with salt and pepper chromatin (arrow head). Second component: goblet cells displaying univacuolated cytoplasm and peripherally compressed nuclei (arrow) (H\&E, 200x).

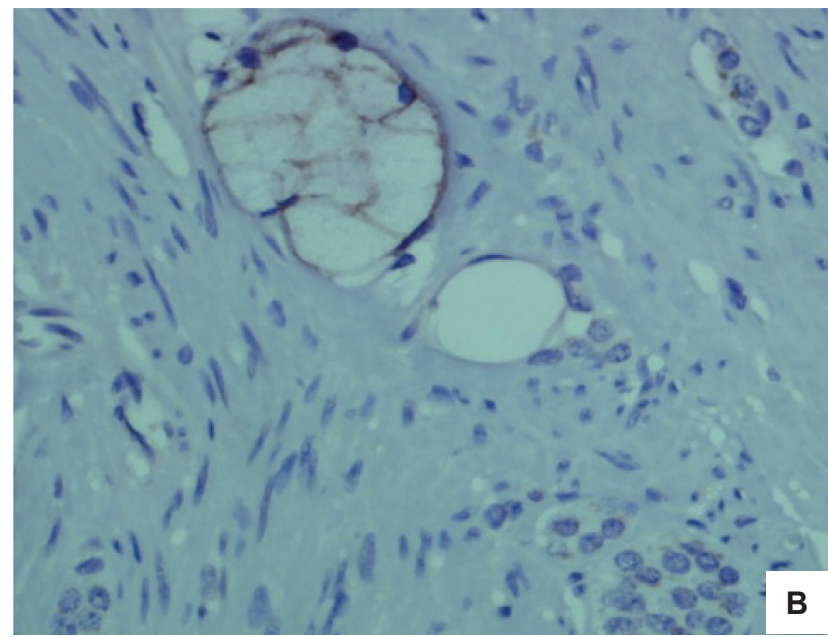

Figure 3. Immunohistochemical findings revealed both components are positive for synaptophysin (A). However, only the goblet cells are positive for CK20 (B) (400x).

\section{DISCUSSION}

Our patient was diagnosed to have Tang's A GCC and underwent an appendectomy followed by right hemicolectomy. Often diagnosed post-operatively during histopathological examinations, GCCs can sometimes be mistaken as a neuroendocrine tumour (NET). The diagnosis is vital as GCCs exhibit more aggressive behaviour compared to the typical appendiceal neuroendocrine tumours (TNET), which are often managed conservatively. ${ }^{3}$ In other words, the overall prognosis of GCCs are poorer compared to NETs, and a more aggressive plan of management and surveillance is essential for these patients.

GCCs are distinctive from the other appendiceal tumours as they have both neuroendocrine differentiation and goblet cell type morphology. The cells stain weakly positive for classical neuroendocrine tumour markers, i.e., chromogranin A and synaptophysin, and they simultaneously produce mucin, pathognomonic for colorectal adenocarcinomas.
The natural history of GCCs is intermediate in its aggressiveness between classical adenocarcinomas and carcinoids, with reported 5-year survival rates of 58\%$83 \%$. Due to its natural history and malignant nature, treatment recommendations are in general similar to adenocarcinomas rather than classical carcinoids, with the role of right hemicolectomy in non-extensive GCCs still being debated.

Staging of GCCs include both the 2010 World Health Organization (WHO) classification for appendix tumours, the 2010 AJCC (TNM classification) staging, and Tang et al., classification. ${ }^{2,4}$ The Tang classification divides the tumours into three distinctive group based on histological features such as degree of atypia, degree of desmoplasia and arrangement of goblet cells.

The cornerstone of treatment for non-metastatic disease is surgical resection, yet the extent of resection is still debatable. Contrary to the TNETs, there is no consensus among the European Neuroendocrine Tumour Society 
(ENETS) and North American Neuroendocrine Tumour Society (NANETS). Despite ENETS's recommendation to proceed with a right hemicolectomy and/or salphingooopherectomy after appendectomy, the evidence is still scarce due to absence of randomized controlled trials. $3,5,6$ A review by Gilmore et al., quoted a meta-analysis of 13 studies by Varisco et al., showing no benefits of right hemicolectomy in patients with localized disease with low grade histology, while other studies also show no benefits of right hemicolectomy in those with small $(<1 \mathrm{~cm})$, localized, low grade tumours without high risk features such as a positive resection margin.?

Retrospective analysis of Surveillance, Epidemiology, and End Results (SEER) data evaluating 3137 patients with appendiceal NETS, showed only statistically significant benefit of right hemicolectomy in appendiceal NETs with signet ring cell adenocarcinoma histology, while there were no significant surgical benefits for right hemicolectomy versus appendectomy for typical NETS, after adjusting for age, stage and histology. ${ }^{6}$ Tang et al., noted in their analysis that tumour of higher grades benefit more with right hemicolectomy, suggesting that tumours $<2 \mathrm{~cm}$, locally advanced stage, positive margins, group B and C histologically, or pT3 and pT4 tumours warrants a right hemicolectomy. ${ }^{4,7}$

Treatment of advanced GCCs mainly includes chemotherapy, but currently, there are no randomized control trials or evidenced-based guidelines. In view of its resemblance to a colon adenocarcinoma, 5-FU based chemotherapies have been suggested despite the scant evidence. Theranostic therapies may not be appropriate, since GCCs are not Ga-DOTATE avid.

Monitoring of recurrence with biochemical investigations (serum CgA or urinary 5-HIAA) are not useful, as these tumours are often non-functional. Contrast CT or MRI scans are sensitive to detect recurrence, whilst the FDG PET/ CT scan is sensitive for advanced GCCs. Ga-DOTATATE PET/CT scans in these patients are often negative as GCCs rarely possess somatostatin 2 receptors. Conventional tumour markers of adenocarcinoma of the colon such as carcinoembryonic antigen (CEA), CA125, CA-19-9, may have better value for surveillance for recurrence. ${ }^{2}$

Overall prognosis of GCCs depends on the staging and histology of the tumour. Tang et al., reported that the 5-years disease-specific survival reduces according to the groups, with 100\% (group A), 36\% (group B), and $0 \%$ (group C) respectively. ${ }^{4}$ Patients with a major adenocarcinoma component $(>50 \%)$ have a poorer prognosis compared with those with minor $(<50 \%)$ component. ${ }^{1}$

\section{CONCLUSION}

GCC tumours are more aggressive than classical neuroendocrine tumours even if they do not exhibit malignant properties of adenocarcinomas. Thus, they should be identified promptly as their further definitive therapy differs from the other gastrointestinal NETs. The cornerstone treatment for GCCs are surgical removal of the tumour, yet the value of right hemicolectomy in localised diseased is still unknown. ${ }^{8}$ To date, there are still no consensus on the follow-up algorithm of GCCs. In the future, collaborative multi-centre studies will be integral in identifying the best treatment and monitoring options.

\section{Ethical Consideration}

Patient consent was obtained before submission of the manuscript.

\section{Statement of Authorship}

All authors certified fulfillment of ICMJE authorship criteria.

\section{Author Disclosure}

The authors declared no conflict of interest.

\section{Funding Source}

None.

\section{References}

1. Taggart MW, Abraham SC, Overman MJ, Mansfield PF, Rashid A. Goblet cell carcinoid tumor, mixed goblet cell carcinoidadenocarcinoma, and adenocarcinoma of the appendix: Comparison of clinicopathologic features and prognosis. Arch Pathol Lab Med. 2015;139(6):782-90. PMID: 26030247. https://doi.org/10.5858/ arpa.2013-0047-OA.

2. Rossi RE, Luong $\mathrm{T}$, Caplin $\mathrm{ME}$, et al. Goblet cell appendiceal tumors--Management dilemmas and long-term outcomes. Surg Oncol. 2015;24(1):47-53. PMID: 25686643. https://doi.org/10.1016/ j.suronc.2015.01.001.

3. Boudreaux JP, Klimstra DS, Hassan MM, et al. The NANETS consensus guideline for the diagnosis and management of neuroendocrine tumors: Well-differentiated neuroendocrine tumors of the jejunum, ileum, appendix, and cecum. Pancreas. 2010;39(6):753-66. PMID: 20664473. https://doi.org/10.1097/MPA.0b013e3181ebb2a5.

4. Tang LH, Shia J, Soslow RA, et al. Pathologic classification and clinical behavior of the spectrum of goblet cell carcinoid tumors of the appendix. Am J Surg Pathol. 2008;32(10):1429-43. PMID: 18685490. https://doi.org/10.1097/PAS.0b013e31817f1816.

5. Pape UF, Perren A, Niederle B, et al. ENETS consensus guidelines for the management of patients with neuroendocrine neoplasms from the jejuno-ileum and the appendix including goblet cell carcinomas. Neuroendocrinology. 2012;95(2):135-56. PMID: 22262080 https://doi.org/10.1159/000335629.

6. Shaib W, Krishna K, Kim S, et al. Appendiceal neuroendocrine, goblet and signet-ring cell tumors: A spectrum of diseases with different patterns of presentation and outcome. Cancer Res Treat 2016;48(2):596-604. PMID: 26044156. PMCID: PMC4843718. https:// doi.org/10.4143/crt.2015.029.

7. Gilmore G, Jensen K, Saligram S, Sachdev TP Arekapudi SR Goblet cell carcinoid of the appendix - diagnostic challenges and treatment updates: A case report and review of the literature. J Med Case Rep. 2018;12(1):125. PMID: 30244681. PMCID: PMC6151924. https://doi.org/10.1186/s13256-018-1789-6.

8. Clift AK, Kornasiewicz O, Drymousis P, et al. Goblet cell carcinomas of the appendix: Rare but aggressive neoplasms with challenging management. 2018;7(2):268-77. PMID: 29335251. PMCID: PMC5801558. https://doi.org/10.1530/EC-17-0311.

Authors are required to accomplish, sign and submit scanned copies of the JAFES Author Form consisting of: (1) Authorship Certification, that authors contributed substantially to the work, that the manuscript has been read and approved by all authors, and that the requirements for authorship have been met by each author; (2) the Author Declaration, that the article represents original material that is not being considered for publication or has not been published or accepted for publication elsewhere, that the article does not infringe or violate any copvrights or intellectual property rights, and that no references have been made to predatory/ suspected predatory journals; (3) the Author Contribution Disclosure, which lists the specific contributions of authors; and (4) the Author Publishing Agreement which retains author copyright, grants publishing and distribution rights to JAFES, and allows JAFES to apply and enforce an Attribution-Non-Commercial Creative Commons user license. Authors are also required to accomplish, sign, and submit the signed ICMJE form for Disclosure of Potential Conflicts of Interest. For original articles, authors are required to submit a scanned copy of the Ethics Review Approval of their research as well as registration in trial registries as appropriate. For manuscripts reporting data from studies involving animals, authors are required to submit a scanned copy of the Institutional Animal Care and Use Committee approval. For Case Reports or Series, and Images in Endocrinology, consent forms, are required for the publication of information about patients; otherwise, appropriate ethical clearance has been obtained from the institutional review board. Articles and any other material published in the JAFES represent the work of the author(s) and should not be construed to reflect the opinions of the Editors or the Publisher. 